\title{
Metal-Free Synthetic Approach to 3-Monosubstituted Unsymmetrical 1,2,4,5-Tetrazines Useful for Bio-orthogonal Reactions
}

\begin{abstract}
Yangyang Qu, François-Xavier Sauvage, Gilles Cla
Abstract: A facile, efficient and metal-free synthetic approach to 3monosubstituted unsymmetrical 1,2,4,5-tetrazines is presented. Dichloromethane (DCM) is for the first time recognized as a novel reagent in the synthetic chemistry of tetrazines. Eleven 3-aryl/alkyl 1,2,4,5-tetrazines were prepared in excellent yields (up to $75 \%$ ) through this novel approach. The mechanism of this new reaction, including the role of DCM in the tetrazine ring formation, has been investigated by $\mathrm{DCM}^{13} \mathrm{C}$ labeling, and is also presented and discussed as well as the photophysical and electrochemical properties.
\end{abstract}

Among other bio-orthogonal reactions, tetrazine click chemistry with strained alkenes or alkynes (also called inverse Electron Demand Diels Alder (iEDDA) reaction) has recently emerged as a powerful tool for biological imaging and detection, cancer targeting, drug delivery and biomaterials science. ${ }^{[1]}$ The synthesis of reactive tetrazine reagents has recently attracted significant interest since the inception of this field. ${ }^{[2]}$ Moreover, as a well-known electron-deficient heterocycle, ${ }^{[3]}$ 1,2,4,5tetrazines have already seen significant use for decades in various application fields such as organic electronics (e.g., OPVs, OFETs), ${ }^{[4]}$ energetic materials,${ }^{[5]}$ coordination chemistry, ${ }^{[6]}$ electrofluorochromism ${ }^{[7]}$ and total synthesis of natural products. ${ }^{[8]}$ Despite the promising application potential of 1,2,4,5-tetrazines, the lack of practical and convenient tetrazine synthetic approaches has hampered their development, especially for click chemistry related applications. ${ }^{[3]}$ Therefore, developing synthetic routes that improve access to tetrazine molecules has recently become very useful, as it not only eases existing tetrazine applications, but also facilitates the emergence of new applications.

Herein we report a facile and efficient synthetic approach to 3monosubstituted unsymmetrical 1,2,4,5-tetrazines, which are well-known to be the most reactive tetrazines for room temperature (RT) click reactions. With our new approach, 3monosubstituted unsymmetrical tetrazines are conveniently prepared in mild conditions from aromatic/alkyl nitrile precursors and dichloromethane (DCM). 11 tetrazine examples were prepared with excellent yields (up to $75 \%$ ) through this novel approach. To the best of our knowledge, this is the first example utilizing DCM as a reactant in the chemistry of tetrazine synthesis. This approach gives improved access to both 3 -aryl and 3-alkyl unsymmetrical 1,2,4,5-tetrazines in high yields, which are expected to be widely utilized in various applications,

[a] Y. Qu, F.-X. Sauvage, Dr. G. Clavier, Prof. F. Miomandre, Prof. P. Audebert

PPSM- CNRS- ENS Paris-Saclay

61 Avenue Président Wilson, 94235 Cachan (France)

E-mail: audebert@ppsm.ens-cachan.fr

Supporting information for this article is given via a link at the end of the document particularly in bio-orthogonal click chemistry.

The classical approach to prepare 1,2,4,5-tetrazines is a twostep procedure starting from the addition of hydrazine to nitrile precursors, followed by oxidation of the resulting 1,2dihydrotetrazine..$^{[9]}$ However, this approach is usually not efficient for unsymmetrical tetrazines. 3-monosubstituted unsymmetrical 1,2,4,5-tetrazines were conventionally prepared from aromatic or alkyl nitrile precursors and formamidine salts, but yields are typically low, between $10 \%$ to $20 \% .^{[10]}$ Recently, Devaraj et al. demonstrated an effective metal-catalyzed one-pot procedure to prepare 3-monosubstituted unsymmetrical 1,2,4,5tetrazines. ${ }^{[1]}$ This approach is currently intensively employed by scientists to prepare tetrazines useful for iEDDA reactions. However, the limitations of this approach are also obvious because it requires excess anhydrous hydrazine as the solvent (usually 50 equivalents to the nitrile precursors). Anhydrous hydrazine is an oily, highly toxic and hazardous reagent, which is commercially limited in several parts of the world, including Europe and China due to safety regulations. As a result, this synthetic procedure is limited to certain labs or companies. In addition, it also requires excess formamidine salts (usually 10 equiv.) and a non-neglectable amount of expensive metal catalysts ( 0.5 equiv.) (Therefore explaining the high prices of commercial clickable tetrazines, usually in the $1,000 \$ / \mathrm{g}$ range). Moreover, the harsh conditions are not compatible with several functional groups such as carbonyls and alkyl halides, which are susceptible to give either nucleophilic addition or reduction. ${ }^{[12]}$ We envisioned developing a simple but also efficient synthetic approach to prepare 3-monosubstituted unsymmetrical 1,2,4,5tetrazines. In our synthetic approach, DCM was selected as a novel starting material instead of formamidine salts, and we found out it exhibits a surprisingly good reactivity and selectivity (Scheme 1). Because of this excellent reactivity and selectivity, only mild reaction conditions are required. The dangerous anhydrous hydrazine can be replaced by the much more stable hydrazine hydrate as a reactant, and ethanol (EtOH) as the solvent, along with 2 equivalents of sulfur. Only 1 equiv. of DCM is needed rather than excess amount (10 equiv.) of formamidine salts. More importantly, no metal catalyst is required in this novel approach. All these factors make our approach simple, economical and efficient.

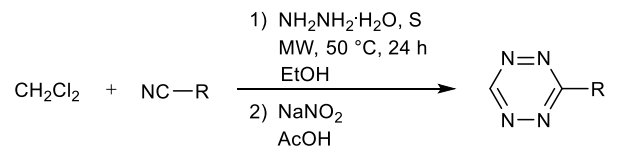

Scheme 1: Synthetic approach for 3-monosubstituted unsymmetrical 1,2,4,5tetrazines.

We optimized the reaction conditions using 4-cyanophenylacetic acid as nitrile precursor to prepare tetrazine $\mathbf{T 1}$, which is a wellknown tetrazine for bio-orthogonal reactions (Table 1). The 
reaction temperature is extremely important to the outcome of this reaction. We used microwave heating throughout, but we have verified that the microwave irradiation was not necessary, and heating in a sealed tube worked as well, though minor changes to the yields did happen. At $80^{\circ} \mathrm{C}$, the reaction can be finished within 6 hours (most of the nitrile precursor consumed, Entry 1). However, considerable amount of side products were also generated, making the purification tedious, along with a moderate yield of $38 \%$. The yield of the reaction is significantly increased by decreasing the temperature to $50{ }^{\circ} \mathrm{C}$ (Entry 3). Using these conditions, an excellent yield of $72 \%$ was obtained. However, further decreasing the temperature decreases the yield again (Entry 4). In addition, (as historically noticed in Pinner reaction ${ }^{[13]}$ ) sulfur addition was also found important in this reaction. As an inducer of the reaction, 1 equiv. of sulfur already leads to a quite reasonable yield, while slight excesses of sulfur can still improve the yield and 2 equiv. of sulfur gives the best yield. The importance of sulfur addition is also important to elucidate the mechanism of the reaction (see later on). When no sulfur is used, most of the nitrile precursor was recovered and only a small amount of product could be isolated (3\%, Entry 5). However, further excess sulfur (4 equiv.) lowers the yield of the reaction probably because of unwanted side reactions (Entry 9).

Table 1: Optimization of the reaction conditions ${ }^{(a)}$
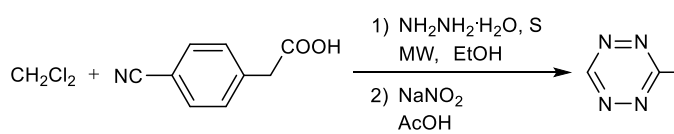

$T$

\begin{tabular}{|l|l|l|l|}
\hline Entry & Temp., time & $\mathbf{S}$ & Yield [\%] $^{(\mathrm{b})}$ \\
\hline $\mathbf{1}$ & $80^{\circ} \mathrm{C}, 6 \mathrm{~h}$ & 2 equiv. & 38 \\
\hline $\mathbf{2}$ & $60^{\circ} \mathrm{C}, 24 \mathrm{~h}$ & 2 equiv. & 61 \\
\hline $\mathbf{3}$ & $50^{\circ} \mathrm{C}, 24 \mathrm{~h}$ & 2 equiv. & 72 \\
\hline $\mathbf{4}$ & $40^{\circ} \mathrm{C}, 24 \mathrm{~h}$ & 2 equiv. & 40 \\
\hline $\mathbf{5}$ & $50^{\circ} \mathrm{C}, 24 \mathrm{~h}$ & 0 equiv. & 3 \\
\hline $\mathbf{6}$ & $50^{\circ} \mathrm{C}, 24 \mathrm{~h}$ & 1 equiv. & 45 \\
\hline $\mathbf{7}$ & $50^{\circ} \mathrm{C}, 24 \mathrm{~h}$ & 1.2 equiv. & 62 \\
\hline $\mathbf{8}$ & $50^{\circ} \mathrm{C}, 24 \mathrm{~h}$ & 1.5 equiv. & 70 \\
\hline $\mathbf{9}$ & $50^{\circ} \mathrm{C}, 24 \mathrm{~h}$ & 4 equiv. & 55 \\
\hline
\end{tabular}

(a) All reactions were carried out on a $0.5 \mathrm{mmol}$ scale in $0.5 \mathrm{ml} \mathrm{EtOH.} 1$ equiv. $\mathrm{DCM}, 1$ equiv. nitrile precursor and 8 equiv. hydrazine hydrate (MW = microwave, equiv. = equivalents). (b) Yields (isolated) based on the nitrile precursor.

To extend the scope of this synthetic approach, a series of 3monosubstituted unsymmetrical 1,2,4,5-tetrazines were prepared employing this approach (Table 2). Several useful unsymmetrical tetrazines T1, T2, T3 and T4 were successfully prepared in good to high yields. Interestingly, when using 4(aminomethyl)benzonitrile hydrochloride as the nitrile precursor, the desired tetrazine with amine functional group was not obtained, instead tetrazine T3 with hydroxy group was obtained in high yield $(71 \%$, Scheme 2), due to the reaction of nitrous acid $\left(\mathrm{HNO}_{2}\right)$ with primary amine during the oxidation step. The yield is even higher compared to using 4(hydroxymethyl)benzonitrile as the nitrile precursor which is commercially-unfriendly. This reaction offers a more efficient and economical pathway to tetrazine T3.

Table 2: Synthesis of 3-monosubstituted unsymmetrical tetrazines T1-T11(a)

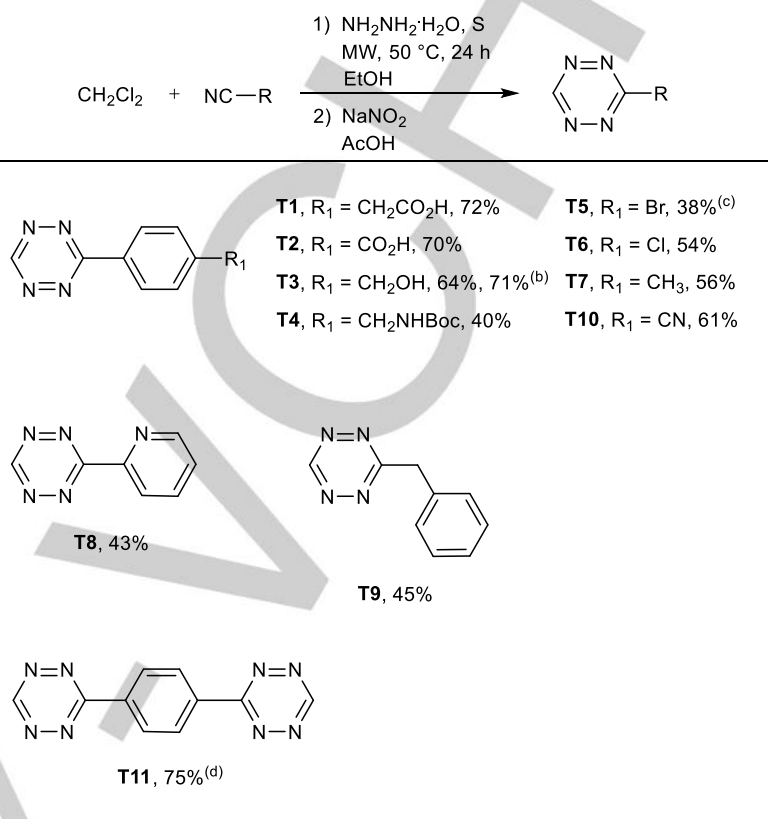

(a) Reactions were carried out on a $1 \mathrm{mmol}$ scale in $1 \mathrm{ml} \mathrm{EtOH}$. Yields (isolated) based on the starting nitrile. 1 equiv. DCM, 1 equiv. nitrile precursor, 2 equiv. sulfur and 8 equiv. hydrazine hydrate (except T11). (b) T3 was alternatively prepared using 4-(aminomethyl)benzonitrile hydrochloride as the nitrile precursor. (c) $0.2 \mathrm{ml}$ DMF as co-solvent. (d) 2 equiv. DCM, 1 equiv. nitrile precursor, 4 equiv. sulfur and 16 equiv. hydrazine hydrate.

Tetrazines T5, T6, T7 and T8 were also easily prepared in moderate to high yields as expected, among which T5 and T6 are useful for further preparation of new photo and/or electroactive molecular materials through coupling reactions, an outcome which is currently under investigation in our group. Importantly, unsymmetrical alkyl tetrazines could also be prepared in high yield by this approach. T9 was directly prepared from the alkyl nitrile precursor benzyl cyanide and DCM in a moderate yield (45\%).

Remarkably, when using 1,4-dicyanobenzene as the nitrile precursor, the two cyano groups can be sequentially converted to tetrazine groups. Therefore, tetrazine T10 and T11 were readily prepared in high yields. Notably, T11 is a di-tetrazine molecule which has two functional sites adapted for click reactions, which could potentially serve as a bifunctional bioorthogonal tetrazine reagent.

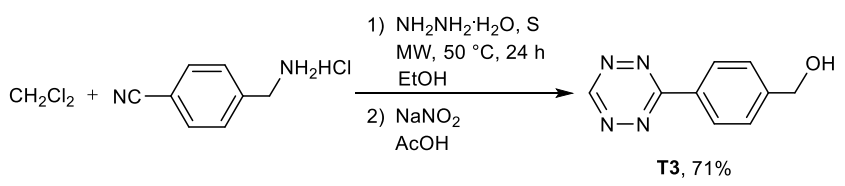

Scheme 2: Synthesis of T3 using 4-(aminomethyl)benzonitrile hydrochloride as the nitrile precursor. 
In order to explore the mechanism of the reaction, the participation of $\mathrm{CH}_{2} \mathrm{Cl}_{2}$ in the tetrazine ring formation was firstly confirmed by using ${ }^{13} \mathrm{CH}_{2} \mathrm{Cl}_{2}$ as the reactant (Scheme 3). The doublet peak at $10.17 \mathrm{ppm}\left({ }^{1} \mathrm{~J}_{\mathrm{CH}}=212.04 \mathrm{~Hz}\right)$ of ${ }^{1} \mathrm{H}$ NMR and the extraordinary high intensity of singlet peak at $157.73 \mathrm{ppm}$ of ${ }^{13} \mathrm{C}$ NMR can be ascribed to the proton and carbon of $\mathrm{H}^{-13} \mathrm{C}$ group, respectively (Figure 1). In addition, the C-3 carbon shows a doublet peak at $166.61 \mathrm{ppm}\left({ }^{3} \mathrm{JCC}_{\mathrm{C}}=29.20 \mathrm{~Hz}\right)$ in ${ }^{13} \mathrm{C}$ NMR (Figure 1 lower), which also indicates the incorporation of ${ }^{13} \mathrm{C}$ into the tetrazine ring of $\mathrm{T} 12$.

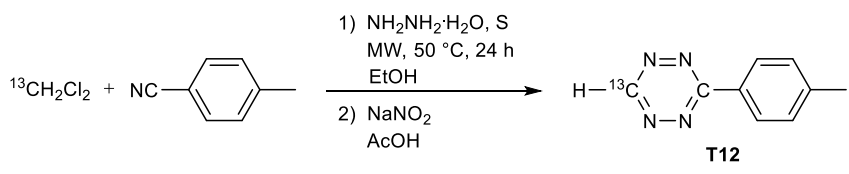

Scheme 3: Synthesis of T12.
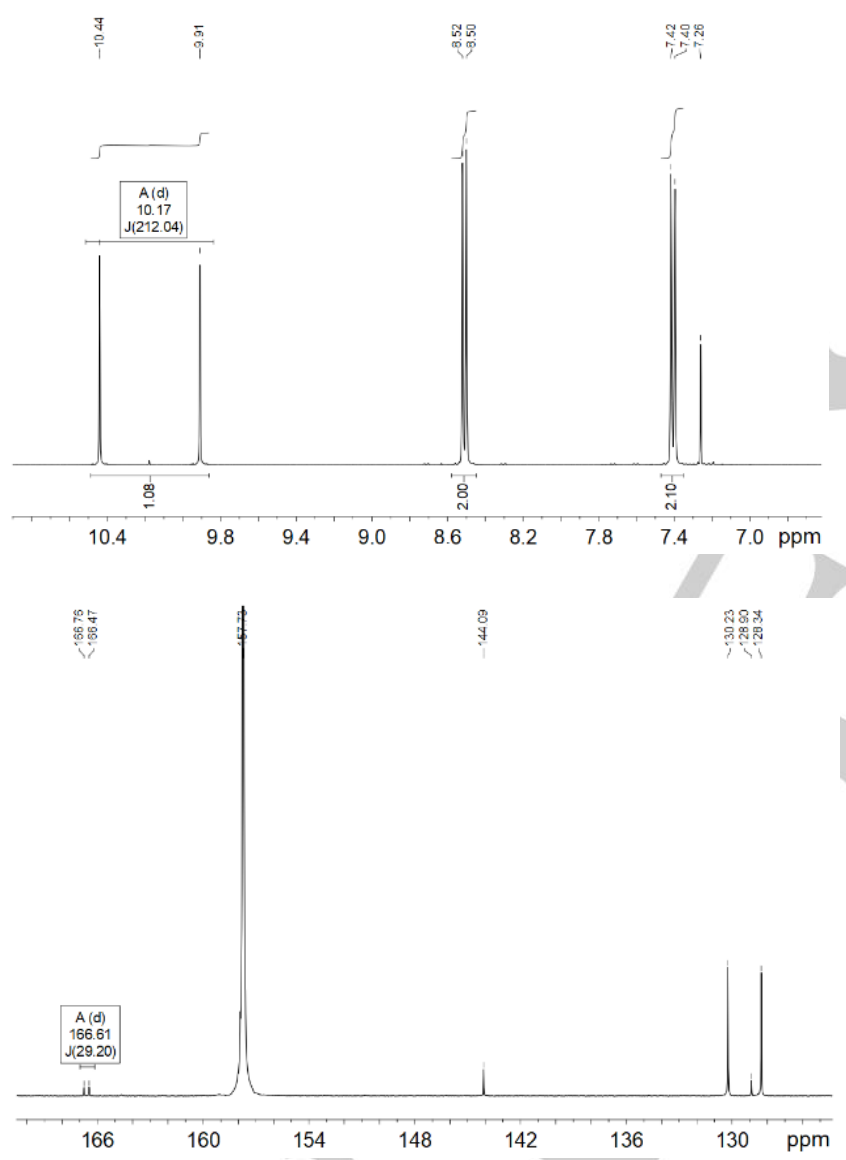

Figure 1: ${ }^{1} \mathrm{H}$ NMR ((upper, $400 \mathrm{MHz}, \mathrm{CDCl}_{3}$ ) and ${ }^{13} \mathrm{C}$ NMR (lower, $100 \mathrm{MHz}$, $\mathrm{CDCl}_{3}$ ) spectra of $\mathbf{T 1 2}$. Only downfield parts of the spectra are shown here to show the characteristic signals clearly (see NMR spectra T12 in supporting information for full spectra).

The formation of 1,2,4,5-tetrazine is commonly agreed as a twostep procedure starting from the nucleophilic attack of nitrile precursor by sulfur activated hydrazine, followed by oxidation of the resulting 1,2-dihydrotetrazine. ${ }^{[3]}$ Since no standard "Pinner" product is observed, with as little as 1 equiv. of DCM added, the first step is very likely addition of the sulfur-activated hydrazine on DCM, followed by a second substitution step, on the second chlorine, rendered more reactive because of the first addition. Since a noticeable amount of product is formed with as little as 1 equiv. of sulfur (despite 2 equiv. improve the yields), and that the dihydrotetrazine is formed before oxidant addition, it is likely that hydrazine alone reacts in this second addition step. Then addition on the nitrile likely occurs, It does not matter which terminal nitrogen attacks at the cyano group, because the following cyclisation step leads to the same ring. Finally, a double elimination occurs, before the final oxidative rearomatization step. Following this reasoning, a possible mechanism for tetrazine ring formation using $\mathrm{CH}_{2} \mathrm{Cl}_{2}$ as a reagent is summarized in Scheme 4.

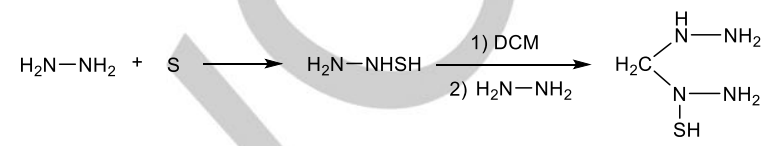

$\mathrm{R}-\mathrm{CN}$

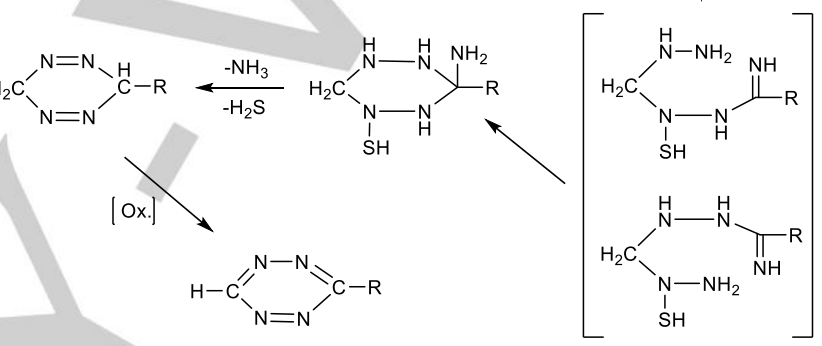

Scheme 4: Proposed mechanism for tetrazine ring formation using $\mathrm{CH}_{2} \mathrm{Cl}_{2}$ as a reagent.

To support more strongly our mechanism, and lead by the idea that actually, the activated hydrazine was implicated in the first addition step only, and not further, we have investigated the influence of intermediate amounts of sulfur. Actually, 1.2 equiv. of sulfur already leads to quite good yieds, and 1.5 equiv. give almost the same high yield than 2 equiv. This demonstrates that sulfur in moderate excess to 1 equiv. is sufficient to the reaction, the excess probably serving to compensate for a nonquantitative formation of the sulfur-hydrazine activated nucleophile (due to the heterogenous reaction). This strongly suggests that only 1 equiv. of activated nucleophile is used at the beginning of the reaction process, 1 equiv. of hydrazine alone being involved afterwards in one of the following steps, as proposed in Scheme 4.

It is worth mentioning, however, that the reaction did not work in a few trials with the more hindered dichloroethane, and seems probably limited to DCM as a synthon, despite our attempts were limited to this dichloroalkane only.

The photophysical data for the T1-T9 series are gathered in Table 3. All compounds present similar features, typical of tetrazine derivatives, namely an absorption band in the visible with a maximum located near $535 \mathrm{~nm}$ and a rather low extinction coefficient. Unfortunately, a very weak emission in the 575-595 $\mathrm{nm}$ range has been recorded for all the series (only T4 exhibits a luminescence quantum yield over 1\%). These poor fluorescence properties were expected since it is known that only tetrazines substituted with heteroatoms are highly emissive. ${ }^{[3]}$ 
Table 3: Absorption and emission maximum wavelengths

\begin{tabular}{|l|l|l|l|}
\hline & $\begin{array}{l}\text { Absorption } \\
\text { wavelength }^{(\mathrm{a})}\end{array}$ & $\begin{array}{l}\text { Emission } \\
\text { wavelength }\end{array}$ & Quantum yield \\
& $(\mathrm{c})$ \\
\hline T1 & $536(300)^{(\mathrm{b})}$ & 595 & $0.17 \%$ \\
\hline T2 & $534(270)$ & - & - \\
\hline T3 & $535(750)$ & 581 & $0.17 \%$ \\
\hline T4 & $535(500)$ & 598 & $0.20 \%$ \\
\hline T5 & $533(580)$ & 577 & $1.40 \%$ \\
\hline T6 & $533(880)$ & 575 & $0.13 \%$ \\
\hline T7 & $535(930)$ & 578 & $0.21 \%$ \\
\hline T9 & $535(400)$ & 587 & $0.19 \%$ \\
\hline
\end{tabular}

(a) Values in $\mathrm{nm}$. (b) The values in brackets are extinction coefficients. (c) Luminescence quantum yields of solutions of compounds T1-T9 in acetonitrile.

The electrochemistry of compounds T1-T9 was studied in solution in acetonitrile. Two types of cyclic voltammetries (CV) are observed:

For non-acidic compounds a nicely reversible $\mathrm{CV}$ is observed (Figure 2, full line).

For acidic compounds (T1-T2), a fully irreversible reduction peak can be seen (Figure 2, dotted line)

All CVs have been compared to a standard (chloroethoxytetrazine) to assess the number of electrons exchanged on one hand and the standard potential on the other hand. Using a standard allows one to get rid of electrode surface issues for the relation between peak current and number of electrons and of reference drift for the determination of standard potential.

The results are summarized in Table 4. All compounds behaving reversibly shows a one-electron reduction as expected, leading to the anion radical which is thus stable at the timescale of cyclic voltammetry. Moreover, the standard potentials for the redox couple formed by tetrazine and its anion radical all lie around $1.35 \mathrm{~V}$ vs. Fc which is similar to tetrazines substituted by donating aromatic groups like pyrrole or thiophene. ${ }^{[3]}$ It can be noticed that T5-T6 have a more positive reduction potential of ca. $+0.1 \mathrm{~V}$ in agreement with the withdrawing character of the halogen substituents born by the tetrazine core. This demonstrates that the redox potentials of these tetrazine derivatives are actually sensitive to the electronic properties of the substituent.

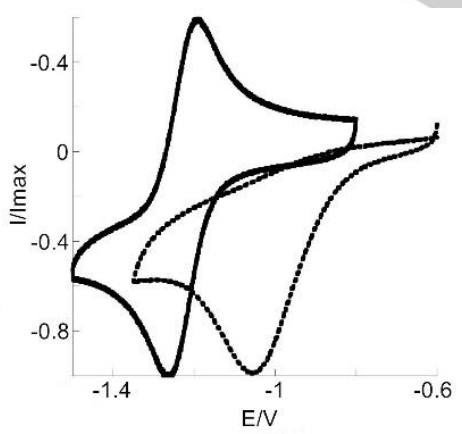

Figure 2: $\mathrm{CV}$ s of $\mathrm{T7}$ (full line) and T1 (dotted line) in acetonitrile (+TBAPF 6 ) on glassy carbon electrode at $0.1 \mathrm{~V} / \mathrm{s}$. Currents are normalized vs. the peak cathodic value for clarity sake.
The two compounds substituted with acidic functions show a non-reversible electrochemical behavior assigned to the rapid protonation of the anion radical. This leads to more positive reduction potential (typically $-1.14 \mathrm{~V}$ for the peak reduction potential of T1) in agreement with an 'EC' mechanism. Besides the number of electrons exchanged which has been found close to 2 is in agreement with a ' $2 \mathrm{H}^{+}+2 \mathrm{e}^{-4}$ mechanism similar to quinone reduction. Typically after the one-electron first reduction and fast protonation, the generated radical is further reduced and the anion protonated.

Table 4: Electrochemical data for T1-T9 ${ }^{(\mathrm{a})}$

\begin{tabular}{|l|l|l|}
\hline & $\begin{array}{l}\text { Standard potential /V } \\
\text { vs. Fc }{ }^{+/ F c}\end{array}$ & $\begin{array}{l}\text { Exp. det. number of } \\
\text { electrons exchanged }\end{array}$ \\
\hline T1 & n.d. ${ }^{(b)}$ & 2.25 \\
\hline T2 & n.d. & 1.88 \\
\hline T3 & -1.33 & 0.97 \\
\hline T4 & -1.35 & 0.98 \\
\hline T5 & -1.23 & 0.89 \\
\hline T6 & -1.24 & 1.12 \\
\hline T7 & -1.32 & 0.95 \\
\hline T9 & -1.35 & 0.97 \\
\hline
\end{tabular}

(a) In acetonitrile (+TBAPF 6 ) on glassy carbon electrode. (b) n.d. = not defined.

In summary, we have discovered a facile, efficient and metalfree synthetic approach to 3-monosubstituted unsymmetrical 1,2,4,5-tetrazines. DCM has been demonstrated to be a novel alternative reagent to formamidine salts which exhibits excellent reactivity and selectivity during the tetrazine ring formation, resulting in high yields and mild reaction conditions in the tetrazine synthesis. 3-monosubstituted unsymmetrical 1,2,4,5tetrazines were thus prepared which are among the most useful reagents in bio-orthogonal click chemistry as well as other application fields (e.g., photo and/or electroactive molecular materials).

\section{Acknowledgements}

The research leading to these results has received funding from the European Union's Horizon 2020 research and innovation programme under the Marie Skłodowska-Curie grant agreement No 674990 (EXCILIGHT).

Keywords: bio-orthogonal reaction $\bullet$ click chemistry $\bullet$ metal-free - synthetic approach • tetrazines

[1] a) H. Wu, S. C. Alexander, S. Jin, N. K. Devaraj, J. Am. Chem. Soc 2016, 138, 11429-11432; b) I. Nikić, G. E. Girona, J. H. Kang, G. Paci, S. Mikhaleva, C. Koehler, N. V. Shymanska, C. V. Santos, D. Spitz, E. A. Lemke, Angew. Chem. Int. Ed. 2016, 55, 16172-16176; c) C. Uttamapinant, J. D. Howe, K. Lang, V. Beranek, L. Davis, M. Mahesh, N. P. Barry, J. W. Chin, J. Am. Chem. Soc. 2015, 137, 4602-4605; d) F. Ehret, H. Wu, S. C. Alexander, N. K. Devaraj, J. Am. Chem. Soc. 2015 137, 8876-8879; e) P. Agarwal, B. J. Beahm, P. Shieh, C. R. Bertozzi, Angew. Chem. Int. Ed. 2015, 54, 11504-11510; f) L. G. Meimetis, J. C. T. Carlson, R. J. Giedt, R. H. Kohler, R. Weissleder, Angew. Chem. Int Ed. 2014, 53, 7531-7534; g) C. Denk, D. Svatunek, T. Filip, T. Wanek, D. Lumpi, J. Fröhlich, C. Kuntner, H. Mikula, Angew. Chem. Int. Ed. 
2014, 53, 9655-9659; h) H. Wu, B. T. Cisneros, C. M. Cole, N. K. Devaraj, J. Am. Chem. Soc. 2014, 136, 17942-17945; i) Z. Li, D. Wang L. Li, S. Pan, Z. Na, C. Y. J. Tan, S. Q. Yao, J. Am. Chem. Soc. 2014, 136, 9990-9998; j) J. C. Carlson, L. G. Meimetis, S. A. Hilderbrand, R. Weissleder, Angew. Chem. Int. Ed. 2013, 52, 6917-6920; k) K. S. Yang, G. Budin, T. Reiner, C. Vinegoni, R. Weissleder, Angew. Chem. Int. Ed. 2012, 51, 6598-6603; I) T. Plass, S. Milles, C. Koehler, J. Szymanski, R. Mueller, M. Wiessler, C. Schultz, E. A. Lemke, Angew. Chem. Int. Ed. 2012, 51, 4166-4170; m) K. Lang, L. Davis, J. Torres-Kolbus, C. J. Chou, A. Deiters, J. W. Chin, Nat. Chem. 2012, 4, 298-304; n) D. S. Liu, A. Tangpeerachaikul, R. Selvaraj, M. T. Taylor, J. M. Fox, A. Y. Ting, J. Am. Chem. Soc. 2012, 134, 792-795; o) M. R. Karver, R. Weissleder, S. A. Hilderbrand, Angew. Chem. Int. Ed. 2012, 51, 920-922; p) J. B. Haun, N. K. Devaraj, S. A. Hilderbrand, H. Lee, R. Weissleder, Nat. Nanotechnol. 2010, 5, 660-665.

[2] a) M. L. Blackman, M. Royzen, J. M. Fox, J. Am. Chem. Soc. 2008,130, 13518-13519; b) N. K. Devaraj, R. Weissleder, S. A. Hilderbrand, Bioconjugate Chem. 2008, 19, 2297-2299.

[3] G. Clavier, P. Audebert, Chem. Rev. 2010, 110, 3299-3314.

[4] a) Z. Li, J. Ding, N. Song, J. Lu, Y. Tao, J. Am. Chem. Soc. 2010, 132, 13160-13161; b) Z. Li, J. Ding, N. Song, X. Du, J. Zhou, J. Lu, Y. Tao, Chem. Mater. 2011, 23, 1977-1984; c) D. K. Hwang, R. R. Dasari, M. Fenoll, V. Alain-Rizzo, A. Dindar, J. W. Shim, N. Deb, C. FuentesHernandez, S. Barlow, D. G. Bucknall, P. Audebert, S. R. Marder, B Kippelen Adv. Mater. 2012, 24, 4445-4450.

[5] D. E. Chavez, D. A. Parrish, L. Mitchell, G. H. Imler, Angew. Chem. Int Ed. 2017, 56, 3575-3578; b) H. Gao, J. M. Shreeve, Chem. Rev., 2011 , $111,7377-7436$.
[6] a) M. Glöckle and W. Kaim, Angew. Chem., Int. Ed. 1999, 38, 30723074; b) W. Kaim, Coord. Chem. Rev. 2002, 230, 127-139; c) J. Yuasa, A. Mitsui and T. Kawai, Chem. Commun., 2011, 47, 5807-5809.

[7] a) Y. Kim, E. Kim, G. Clavier, P. Audebert, Chem. Commun. 2006 3612-3614; b) F. Miomandre, R. Meallet-Renault, J. J. Vachon, R. B. Pansu, P. Audebert, Chem. Commun. 2008, 16, 1913-1915; c) C. Quinton, V. Alain-Rizzo, C. Dumas-Verdes, G. Clavier, F. Miomandre, P. Audebert, Eur. J. Org. Chem. 2012, 7, 1394-1403; d) P. Audebert, F. Miomandre, Chem. Sci. 2013, 4, 575-584.

[8] a) D. L. Boger, J. Hong, J. Am. Chem. Soc. 2001, 123, 8515-8519; b) A. Hamasaki, J. M. Zimpleman, I. Hwang, D. L. Boger, J. Am. Chem. Soc. 2005, 127, 10767-10770.

[9] a) K. A. Hofmann, O. Ehrhart, Ber. Dtsch. Chem. Ges. 1912, 45, $2731-$ 2740; b) T. Curtius, A. Hess, J. Prakt. Chem. 1930, 125, 40-53.

[10] M. R. Karver, R. Weissleder, S. A. Hilderbrand, Bioconjugate Chem. 2011, 22, 2263-2270.

[11] J. Yang, M. R. Karver, W. Li, S. Sahu, N. K. Devaraj, Angew. Chem. Int. Ed. 2012, 51, 5222-5225.

[12] H. Wu, J. Yang, J. Seckute, N. K. Devaraj, Angew. Chem. Int. Ed. 2014, 53, 5805-5809.

[13] a) M. O. Abdel-Rahman, M. A. Kira, M. N. Tolba, Tetrahedron Lett., 1968, 3871-3872; b) R. N. Butler, F. L. Scott, R. D. Scott, J. Chem Soc. C, 1970, 2510-2512; c) P. Audebert, S. Sadki, F. Miomandre, G Clavier, M. C. Vernières, M. Saoud, P. Hapiot, New J. Chem. 2004, 28 387-392. 
Entry for the Table of Contents (Please choose one layout)

Layout 2:

\section{COMMUNICATION}

$$
\begin{aligned}
& \text { 1) } \mathrm{NH}_{2} \mathrm{NH}_{2} \cdot \mathrm{H}_{2} \mathrm{O}, \mathrm{S} \\
& \mathrm{CH}_{2} \mathrm{Cl}_{2}+\mathrm{NC}-\mathrm{R} \underset{\substack{\mathrm{NaNO} \\
\mathrm{AcOH}}}{\mathrm{EtOH}} \rightarrow
\end{aligned}
$$

Only dichloromethane, sulfur and hydrazine, no metal catalyst

A facile, efficient and metal-free synthetic approach to 3-monosubstituted unsymmetrical 1,2,4,5-tetrazines is presented. Dichloromethane (DCM) is for the first time recognized as a novel reagent in the chemistry of tetrazine synthesis. 11 tetrazine examples were prepared with excellent yields (up to $75 \%$ ) through this novel approach. The role of DCM in the tetrazine ring formation, has been investigated by $\mathrm{DCM}^{13} \mathrm{C}$ labeling.
Y. Qu, F.-X. Sauvage, G. Clavier, F. Miomandre, P. Audebert*

Page No. - Page No.

Novel Metal-Free Synthetic Approach to 3-Monosubstituted Unsymmetrical 1,2,4,5-Tetrazines Useful for Bioorthogonal Reactions

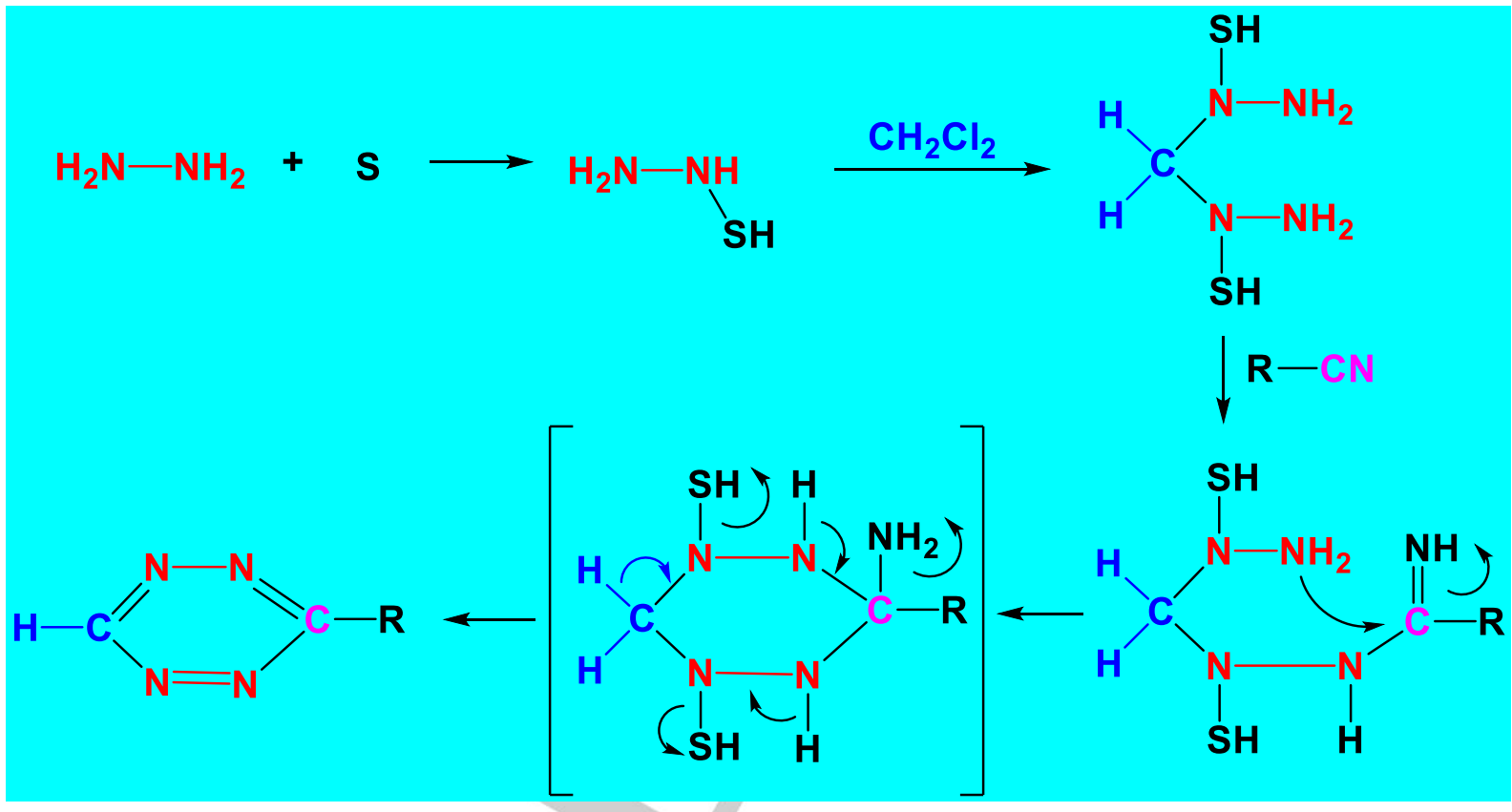

Graphical abstract. 